BULL. AUSTRAL. MATH. SOC.

VOL. $22(1980), 479-480$.

\title{
OPERATOR SEMISTABLE PROBABILITY MEASURES \\ ON A HILBERT SPACE: CORRIGENDA
}

\author{
R.G. LAHA aNd V.K. ROHATg I
}

A faulty typescript of [2] was, regrettably, submitted. The following changes should be made:

Page 398, line 7: replace $A \in G$ with $A \in L$.

Page 398, line 12: replace $A \in G$ with $A \in L$.

Page 400, line 7: replace "It follows ... $y \in H$." with

Since $\hat{\stackrel{P}{P}}(0)=1$ and $\hat{\stackrel{\rho}{P}}$ is continuous on $H$, there exists a $\delta>0$ such that $\hat{\stackrel{P}{P}}(y) \neq 0$ for $\|y\|<\delta$. It then follows from the above relation that $\hat{\hat{Q}}\left(A_{n}^{*} y\right) \rightarrow 1$, as $n \rightarrow \infty$ for $\|y\|<\delta$.

Page 400, line 12: replace "From Proposition 7.4.2" with "From the corollary to Proposition 7.4.1 and from Proposition 7.4.2".

Page 401, immediately before Lemma 2, add the following:

COROLLARY. Let $P \in P$ be a full operator semistable measure. Then $P$ is infinitely divisible.

The proof follows immediately from Lemma 1 and the limiting property of sums of independent $H$-valued random variables satisfying the uniformly asymptotically negligible condition (see [1], page 515).

As an immediate consequence of the above corollary, we note that $\hat{P}(y) \neq 0$ for all $y \in H$.

Page 402: replace last 3 lines with

Received 30 October 1980. 
Moreover, we can verify after some computation that

$$
P=\lim _{n \rightarrow \infty}\left(A^{n} P\right)^{k}{ }^{n} * \delta_{b_{n}^{\prime}}=\lim _{n \rightarrow \infty} A^{n_{P}}{ }^{k} n^{n} \delta_{b_{n}^{\prime}}
$$

where $b_{n}^{\prime}=b_{n} k_{n} / \gamma^{n} \in H$. Hence it follows that $P$ is operator semistable.

Page 403, line 3: replace "Lemma 1 " with "the corollary to Lemma I".

Pages 403 and 404: replace "In view of (10), .. of Lemma 3" with

In view of (10) we have, for every $m \geq 1$,

$$
\begin{aligned}
P & =\lim _{n \rightarrow \infty} A_{m m^{Q^{m}} * \delta_{x_{m n}}} \\
& =\lim _{n \rightarrow \infty} A_{m n} A_{n}^{-1} A_{n} Q^{m n} * \delta_{x_{m}} \\
& =\lim _{n \rightarrow \infty}\left(A_{m n} A^{-1}\right)\left(A_{n} Q^{n} * \delta_{x_{n}}\right)^{m} * \delta_{x_{m n}-m x_{n}} .
\end{aligned}
$$

In view of condition (3), $\left\{A_{m n} A_{n}^{-1}\right\}$ is compact. Let $C_{m} \in G$ be a limit point of the sequence. Passing to the limit through a subsequence if necessary we obtain

$$
P=c_{m} P^{m} * \delta_{a_{m}}
$$

for some $a_{m} \in H$ and for every $m \geq 1$. This completes the proof of Lemma 3 .

Page 404, line 9: replace "In view of Lemma 1 " with "In view of the corollary to Lemma $I^{\prime \prime}$.

\section{References}

[1] R.G. Laha and V.K. Rohatgi, Probability theory (John Wiley \& Sons, New York, Brisbane, Toronto, 1979).

[2] R.G. Laha and V.K. Rohatgi, "Operator semistable probability measures on a Hilbert space", Bull. Austral. Math. Soc. 22 (1980), $397-406$. 